\title{
EXTRACCIÓN Y COMPARACIÓN DE LA QUITINA OBTENIDA A PARTIR DEL CAPARAZON DE Callinectes sapidus Y Penaeus vannameis
}

\section{EXTRACTION AND QUANTITATIVE COMPARISON OF CHITIN OBTAINED FROM THE SHELLOF Callinectes sapidus AND Penaeus vannamei}

\author{
Israel Barros ${ }^{1}$, Luis Guzmán ${ }^{2}$, Arnulfo Tarón ${ }^{3}$
}

\begin{abstract}
${ }^{1}$ Químico Farmacéutico. M.Sc. Ciencia y Tecnología de Alimentos Docente Universidad del Atlántico, Facultad de Química y Farmacia, Km 7 Antigua vía Puerto Colombia, Barranquilla, Colombia. e-mail: israelbarrosp@mail.uniatlantico.edu.co; ${ }^{2}$ Ing. Alimentos. Esp. Ingeniería Sanitaria y Ambiental. Docente Universidad de Cartagena, Programa Ingeniería de Alimentos, Universidad de Cartagena, Facultad de Ingeniería, Campus Piedra de Bolívar. Cartagena-Bolívar, Colombia. e-mail: lguzmanc1@unicartagena.edu.co; autor para correspondencia ${ }^{3}$ Químico Farmacéutico. M.Sc. en Biotecnología. Docente Universidad de Cartagena, Programa Ingeniería de Alimentos, Universidad de Cartagena, Facultad de Ingeniería, Campus Piedra de Bolívar. Cartagena-Bolívar, Colombia. e-mail: atarond@unicartagena.edu.co
\end{abstract}

Rev. U.D.C.A Act. \& Div. Cient. 18(1): 227-234, Enero-Junio, 2015

\section{RESUMEN}

La quitina es el polisacárido natural más abundante en la tierra después de la celulosa y se puede obtener a partir del caparazón de artrópodos. El aumento de la producción de camarón de cultivo ha dado lugar a una mayor cantidad de residuos, lo que plantea problemas ambientales, al generar enormes cantidades de caparazones, las cuales, son abandonadas como residuos. El objetivo de esta investigación fue realizar una comparación cuantitativa de la quitina presente en los caparazones de los artrópodos, utilizando técnicas de caracterización, como espectroscopia infrarroja, valoración potenciométrica y el método Kjeldahl, que hicieron posible evaluar la calidad de la quitina obtenida. Los espectros IR presentaron concordancia con las bandas de quitina del estándar y la quitina extraída del caparazón de jaiba y camarón, con respecto a las señales que aparecen a $3258 \mathrm{~cm}^{-1}$ para grupos $\mathrm{OH}$; a $2877 \mathrm{~cm}^{-1}$ para grupos metílicos; a1654 $\mathrm{cm}^{-1}$ hasta $1069 \mathrm{~cm}^{-1}$ de las vibraciones de amidas I, II, y III y aminas aromáticas y de $1010 \mathrm{~cm}^{-1}$ a $894 \mathrm{~cm}^{-1}$, de los grupos $\mathrm{OH}$ y enlaces $\alpha 1,4$ de este polisacárido.

Palabras clave: Quitina, grado de acetilación, FTIR, jaiba, camarón

\section{SUMMARY}

Chitin is on earth the most abundant natural polysaccharide after cellulose and can be obtained from the shell of arthropods. The increasing production of farmed shrimps has led to a greater amount of waste, which poses environmental problems generated by the huge amounts of shells which are left as garbage. The aim of this research was to assess a quantitative comparison of chitin present in the shell of arthropods using characterization techniques such as infrared spectroscopy, potentiometric titration and the Kjeldahl method, which makes it possible to assess the quality of chitosan obtained. The IR spectra showed bands consistent with the standard chitin and the chitin extracted from crab and shrimp shells with respect to the signals appearing at $3258 \mathrm{~cm}^{-1}$ to $\mathrm{OH}$ groups, at $2877 \mathrm{~cm}^{-1}$ for methyl groups, at $1654 \mathrm{~cm}^{-1}$ to $1069 \mathrm{~cm}^{-1}$ vibration Amide I, II, and III and aromatic amines and $1010 \mathrm{~cm}^{-1}$ to $894 \mathrm{~cm}^{-1}$ of the $\mathrm{OH}$ groups and $\alpha 1,4$ linkages of this polysaccharide

Key words: Chitin, degree of acetylation, FTIR, crab, shrimp.

\section{INTRODUCCIÓN}

En los últimos años, los polímeros naturales han recibido una mayor atención como alternativa a los polímeros sintéticos, con el fin de combinar la fabricación de productos manufacturados, con la protección del medio ambiente, la reducción de los costos de materiales y el reciclado de residuos (Ruiz et al. 2013). El aumento de la producción de camarón de cultivo ha dado lugar a una mayor cantidad de residuos, lo que plantea nuevos problemas ambientales, al generar enormes cantidades de exoesqueletos de cangrejo y de camarón (Teli \& Sheikh, 2012; Cahú et al. 2012). La carne del camarón es separada de los caparazones, 
mientras que los desechos del procesamiento, se componen principalmente del cefalotórax, caparazón y en conjunto, representan entre el 50 y $70 \%$ de todo el camarón (Cahú et al. 2012).

Cada año, grandes cantidades de residuos de jaiba y camarón son vertidos al medio ambiente; esta cantidad de residuos sólidos ocasionan un impacto ambiental negativo. Estos residuos sólidos marinos tienen un gran potencial de aplicación en diferentes industrias; un ejemplo de esta, son la industria farmacéutica y de los alimentos, por mencionar algunas. En el caso de los residuos sólidos de jaiba y camarón, pueden servir como materia prima para la obtención de quitina de su caparazón, logrando darle un tratamiento a estos residuales y conseguir disminuir riesgos de contaminación ambiental. Los residuos generados por la industria de camarón, en el mundo, arrojan toneladas de desechos al mar sin ningún tipo de control, generando con esto un desequilibrio ecológico.

Hastaahora, los caparazonesnosehanutilizadoextensamente, aunque durante los últimos años, una fracción limitada es procesada para ser utilizada como aditivo alimentario en comidas para camarones o se ha usado como materia prima, para la producción de quitina (Rødde et al. 2008). Estos se componen principalmente, de quitina, carbonato de calcio, proteínas, de lípidos y de pigmentos (Mohammed et al. 2013). Estos tres componentes existen estrechamente asociados y se calculan, en cerda de un $90 \%$ del peso seco del caparazón (Rødde et al. 2008). Investigaciones realizadas recientemente, utilizando los caparazones del camarón como materia prima, han logrado obtener quitina y quitosano, para su posible aplicación en revestimiento de alimentos, la eliminación de iones metálicos, anti - tumor, hemostático, anticoagulante, bacteriostático, suturas, piel artificial, en la reconstrucción de los huesos, administración controlada de fármacos y como material de encapsulación (Cahú et al. 2012).

La quitina es el polisacárido natural más abundante en la tierra después de la celulosa y se puede obtener a partir del caparazón de crustáceos marinos, como cangrejos, langostas, camarones y krill (Teli et al. 2012). Estructuralmente, es un polisacárido lineal, que contiene unidades repetidas de $\beta$-(1-4)-2-deoxi-2-acetamido-D glucopiranosas (Goodrich \& Winter, 2007); es blanco, duro e inelástico, presenta baja solubilidad, inmunogenicidad y reactividad química. De ella se puede obtener quitosano, a través de un proceso químico de N-desacetilación (Hernández et al. 2009; Cahú et al. 2012). En investigaciones anteriores, se logró obtener quitosano, a partir de quitina, con un porcentaje de desacetilación, cercano al 75\% (Masum et al. 2011).
El objetivo de esta investigación fue realizar una comparación cuantitativa de la quitina presente en el caparazón de la Jaiba (Callinectes sapidus) y del camarón (Penaeus vannameis) y la utilización de técnicas de caracterización, como espectroscopia infrarroja, valoración potenciométrica y el método Kjeldahl, que hicieron posible cuantificar la calidad de la quitina obtenida. El porcentaje de ceniza, de humedad y de materia insoluble, también fue determinado para verificar su grado de purificación

\section{MATERIALES Y MÉTODOS}

Materia prima. La quitina utilizada en este trabajo fue extraída de las caparazones de jaiba (C. sapidus) y de camarón ( $P$. vannameis), recolectadas en la isla del Rosario de la Ciénaga Grande de Santa Marta, Colombia. La cantidad de muestra de cada especie fue de $400 \mathrm{~g}$ de caparazón de jaiba y caparazón de camarón. Se utilizó quitina comercial (3928086-9), proporcionada por MP Biomedicals LLC Francia.

Obtención de quitina. Los caparazones se lavaron con abundante agua, para eliminar restos orgánicos que pudieran estar presentes y, posteriormente, se secaron al sol. Una vez secos, se sometieron a un proceso de molienda, utilizando un molino doméstico y tamizado hasta obtener un tamaño de partícula menor de $1 \mathrm{~mm}$. Los caparazones de jaiba y camarón, una vez molidos y tamizados por separados, son sometidos a un proceso de despigmentación química, empleando el método propuesto por Meyer \& Blight (1981), con mezcla de éter de petróleo, agua y acetona en la proporción 15:10:75. Para ello, se colocó la harina en un matraz, provisto de agitación por dos horas, a temperatura ambiente; posteriormente, se procedió a filtrar el producto en un embudo Büchner y se secó en una estufa eléctrica tipo Brabender a $50^{\circ} \mathrm{C}$ durante seis horas.

El producto obtenido en la fase anterior, se sometió a desmineralización, siguiendo el método sugerido por Teli et al. (2012), Hernández et al. (2009) y Al-Sagheer et al. (2009); luego, se pesó una cantidad del polvo del crustáceo y se colocó en un matraz, con una solución de $\mathrm{HCl} 1 \mathrm{M}$, en una relación sólido-líquido de $1: 10$, a una temperatura de $30^{\circ} \mathrm{C}$, durante tres horas. El material, se filtró en un embudo de Büchner sobre papel de filtro Whatman $N^{\circ} 1$ y se lavó hasta la neutralidad, con agua destilada; el sobrenadante, se guardó para análisis de los minerales Ca y Mg. La desproteinización química de la muestra, se realizó con una solución de $\mathrm{NaOH} 1 \mathrm{M}$, a una temperatura de $65^{\circ} \mathrm{C}$ y reflujo durante tres horas, con agitación constante, para asegurar una completa desproteinización. La relación utilizada de material: $\mathrm{NaOH}$ fue de 1:15. El producto obtenido se purificó, por filtración en embudo Büchner, realizando lavados con agua destilada, hasta obtener un $\mathrm{pH}$ neutro. 
Composición química de la quitina. Se establecen las características fisicoquímicas de la quitina según lo descrito en la USP 30 del 2007, que determina, a través del espectro infrarrojo y la valoración potenciométrica, el grado de acetilación.

Espectroscopia infrarroja (FT-IR). Se utilizó para identificar estructuralmente, la quitina y su grado de acetilación, siguiendo la técnica sugerida por Brugnerotto et al. (2001). Se emplea un espectrofotómetro IR Perkin-Elmer, modelo 1600 con transformada de Fourier, en un intervalo de frecuencia de $650-4400 \mathrm{~cm}^{-1}$. Las muestras se prepararon sobre pastillas de $\mathrm{KBr}$ (1mg en 100mg de $\mathrm{KBr}$ ) y se estabilizaron, a una humedad relativa controlada. Se determinaron las absorbancias a $1655 \mathrm{~cm}^{-1}$, como medida del contenido del grupo $\mathrm{N}$ - acetilo, y $3450 \mathrm{~cm}^{-1}$, correspondiente a una señal del grupo hidroxilo.

Grado de Acetilación. El método consiste en correlacionar las intensidades de absorbancia de dos bandas determinadas: una tomada como testigo o referencia y, la otra, obtenida experimentalmente; se aplica el método descrito por Domszy \& Roberts (1985). Para la determinación del grado de acetilación por espectroscopia de infrarrojo, donde relacionan las absorbancias obtenidas a las bandas de $1655 \mathrm{~cm}^{-1}$ para la amida I y $3450 \mathrm{~cm}^{-1}$, para grupos hidroxilos, descritos en la ecuación 1 :

$$
\begin{aligned}
& \text { Grado de acetilación }(D A)=\left(A_{1655 \mathrm{~cm}^{-1}} / \mathrm{A}_{3450 \mathrm{~cm}^{-1}}\right) \\
& x(100 / 1.33)
\end{aligned}
$$

Determinación de grupos aminos libres. El porcentaje de grupos aminos libres, se determinó por volumetría potenciométrica, según lo reportado por Jiang et al. (2003) e Hidalgo et al. (2008). Se disolvió $1.5 \mathrm{~g}$ de cada quitina extraída de las diferentes especies por separado, en $25 \mathrm{~mL}$ de $\mathrm{HCl} 0,1 \mathrm{M}$ y se valoró con $\mathrm{NaOH} 0,1 \mathrm{~N}$. Se adicionan volúmenes de base de $2,0 \mathrm{~mL}, 1,0 \mathrm{~mL} ; 0,5 \mathrm{~mL}$ y $0,2 \mathrm{~mL}$ con agitación sucesiva y medición de $\mathrm{pH}$ en cada punto, utilizando un potenciómetro Hanna Instrument Modelo 93140 calibrado, con soluciones de pH 7,01; 4,01 y 10,1. Cada titulación, se realizó por triplicado y con los datos obtenidos, se construyeron las curvas del volumen de $\mathrm{NaOH}$ $0,1 \mathrm{~N}$ añadido contra $\mathrm{pH}$.

Residuos de ignición. El ensayo fue establecido por separado para la quitina extraída del caparazón de jaiba y camarón, utilizando $2 \mathrm{~g}$ de muestra, la cual, fue incinerada, siguiendo el método A.O.A.C. 923.03 (2005). Inicialmente, se procedió a pre calcinar la muestra hasta total carbonización y desaparición de humo blanco, para finalmente incinerar a $550^{\circ} \mathrm{C}$, hasta cenizas blancas o grisáceas. El crisol se enfrío en una desecadora y se pesó en una balanza analítica (OHAUS Adventurer AR3130), repitiéndose esta operación hasta peso constante. Los resultados se expresaron sobre la base de tres replicas.

Contenido de nitrógeno. La determinación se realizó por el método de Kjeldhal en un equipo marca Buchí, dotado de unidad de digestión, scrubber, sistema de destilación y titulador. Para la valoración, se utilizó disolución de ácido sulfúrico $0,01 \mathrm{~N}$ hasta cambio de coloración de verde a azul. El ensayo se realizó por triplicado.

Pérdida por desecación y sólidos totales. Se pesaron, aproximadamente, $2 \mathrm{~g}$ de la muestra, en la balanza analítica (Ohaus Adventurer AR3130), y se mantuvo en estufa de secado Memmert UFE 600 a $105^{\circ} \mathrm{C}$, hasta peso constante. El cálculo, se realizó por triplicado para cada muestra de quitina extraída del caparazón de jaiba y camarón.

Diseño de experimento. En la investigación, se manejó un diseño experimental totalmente aleatorio. Las determinaciones, se efectuaron por triplicado y los resultados expresados, como la media de la desviación estándar. Se utilizó el programa estadístico Statgraphics Centurión XVI.I. Para el análisis de los datos, se aplicó un t-student, fijándose el nivel de significancia a $p<0,05$.

\section{RESULTADOS Y DISCUSIÓN}

Espectroscopia infrarroja. Como se observa en la figura 1 , existe correspondencia entre las bandas asignables a los grupos funcionales de los espectros IR del estándar de quitina con las muestras de jaiba y camarón. Al analizar los espectros IR obtenidos en todos los casos, se puede apreciar una banda ancha de mediana intensidad, asignada al $\mathrm{OH}$, que aparece en $3258 \mathrm{~cm}^{-1}$. En el intervalo entre $2960 \mathrm{~cm}^{-1}$ y $1880 \mathrm{~cm}^{-1}$, presentado en los espectros, a $2877 \mathrm{~cm}^{-1}$, se determinaron unas bandas de mediana intensidad características de C-H simétricos y anti simétricos de grupos metílicos y metilénicos. A $1654 \mathrm{~cm}^{-1}, 1618 \mathrm{~cm}^{-1}, 1560 \mathrm{~cm}^{-1}, 1376 \mathrm{~cm}^{-1}$, $1308 \mathrm{~cm}^{-1}, 1152 \mathrm{~cm}^{-1}$ y $1069 \mathrm{~cm}^{-1}$ aparecen bandas asignadas a las vibraciones de las amidas I, II, y III, respectivamente y aminas aromáticas, donde la primera corresponde al $\mathrm{C}=\mathrm{O}$ y las dos restantes, al $\mathrm{NH}$; todas son bandas agudas de mediana intensidad, a excepción de la que está ubicada a $1069 \mathrm{~cm}^{-1}$ donde se encuentran las amidas III. A $1010 \mathrm{~cm}^{-1}$, están las bandas largas de mayor intensidad, para grupos $\mathrm{OH}$, ya que las señales que aparecen a los $951 \mathrm{~cm}^{-1}$ y $894 \mathrm{~cm}^{-1}$ son bandas agudas de mediana intensidad, pertenecientes al enlace $\alpha(1,4)$, que corresponde a este tipo de polisacárido, concordando con la banda ancha, entre $3259 \mathrm{~cm}^{-1}$, asignada al grupo $\mathrm{OH}$ del estándar del espectro de quitina.

Con la espectroscopia infrarroja, se comprobó la existencia de grupos funcionales reportados en el estándar de quitina, coincidiendo con lo estipulado en cada espectro de las muestras de camarón y jaiba. 


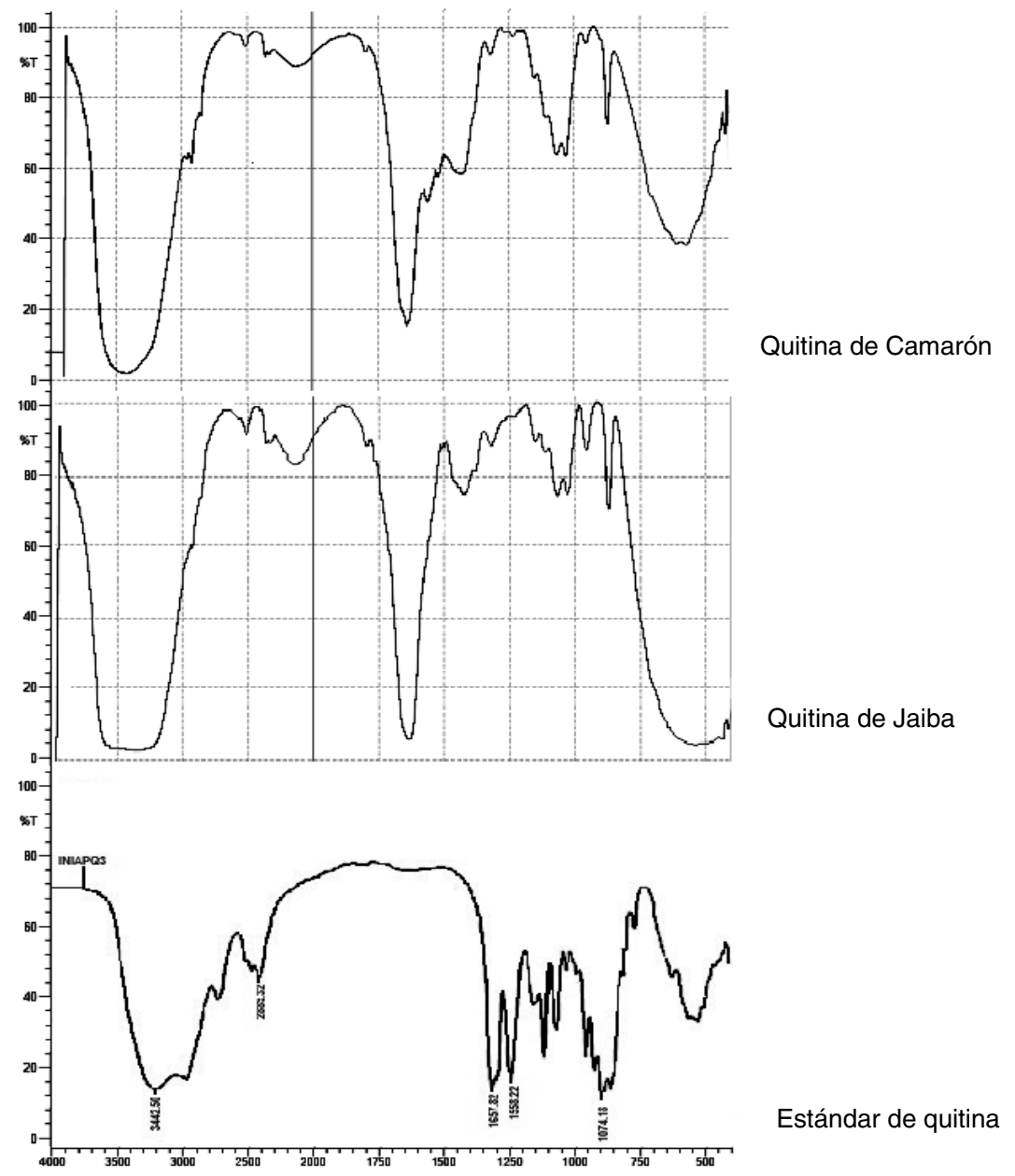

Figura 1. Espectros FTIR de la quitina de jaiba, camarón y estándar.

Grado de Acetilación. Se determinó utilizando lo descrito por Domszy \& Roberts. (1985):

Estándar de quitina

A $3259 \mathrm{~cm}^{1}=96,7 \%$ T, A $1618 \mathrm{~cm}^{1}=95,5 \% \mathrm{~T} ; \mathrm{GA}=74,97 \%$

Muestra de Jaiba (quitina).

A $3261 \mathrm{~cm}^{1}=99,2 \% \mathrm{~T} ;$ A $1618 \mathrm{~cm}^{1}=98,6 \% \mathrm{~T} ; \mathrm{GA}=75,078 \%$ Muestra de camarón (quitina).

A $3258 \mathrm{~cm}^{-1}=98,2 \% \mathrm{~T}$; A $1620 \mathrm{~cm}^{1}=97 \% \mathrm{~T} ; \mathrm{GA}=74,97 \%$

Se observa que los resultados del estándar de quitina con las muestras extraídas de quitina del caparazón de Jaiba y camarón presentan valores similares en el grado de acetilación, lo que comprueba la similitud de las bandas en el infrarrojo.

Grupos aminos libres. En la figura 2, se observa similitud de los gráficos pertenecientes a las muestras de quitina extraída del caparazón de jaiba y camarón, por aplicación de la volumetría potenciométrica, que sirven para determinar los valores de $\Delta \mathrm{pH}$ y $\Delta \mathrm{mL}$, que ayudan a conformar el grafico de la primera derivada, que tiene importancia en la obtención de los valores de los volúmenes de $\mathrm{NaOH}$, requeridos para neutralizar los ácidos libres y establecer la cantidad de acido que protona los grupos aminos de la quitina; la concentración de éstos se determinó utilizando la ecuación (2):

$$
\% \mathrm{NH} 2=45,29(\mathrm{y}-\mathrm{x}) \times \mathrm{F} / \mathrm{W}
$$

Donde $y$ es el punto de inflexión mayor, $x$ punto de inflexión menor, ambos expresados como volumen, $F$ es la molaridad de la solución de $\mathrm{NaOH}, W$ el peso en gramos de cada muestra y 45,29 es un valor relacionado con el peso equivalente de la quitina, cuyo peso molecular promedio es de $1,75 \times 10^{6} \mathrm{~g} /$ mol. El análisis de la volumetría potenciometríca, se realizó por triplicado y los resultados se presentan en la tabla 1 . 
Para ambas muestras, se observa que los volúmenes utilizados para alcanzar el punto final en la titulación dan como resultado un porcentaje del grupo amino, de $24,15 \%$ y $24,76 \%$, equivalentes a un grado de acetilación de 74,97 y 75,08, los cuales, se corresponden con el grado de acetilación del estándar de quitina comercial. Los valores obtenidos para la quitina de jaiba y de camarón son similares a los reportados por Abdou et al. (2008).

Propiedades fisicoquímicas de la quitina. La evaluación de las condiciones del proceso de obtención de la quitina y sus efectos sobre las propiedades físico-químicas, se muestran en la tabla 2.

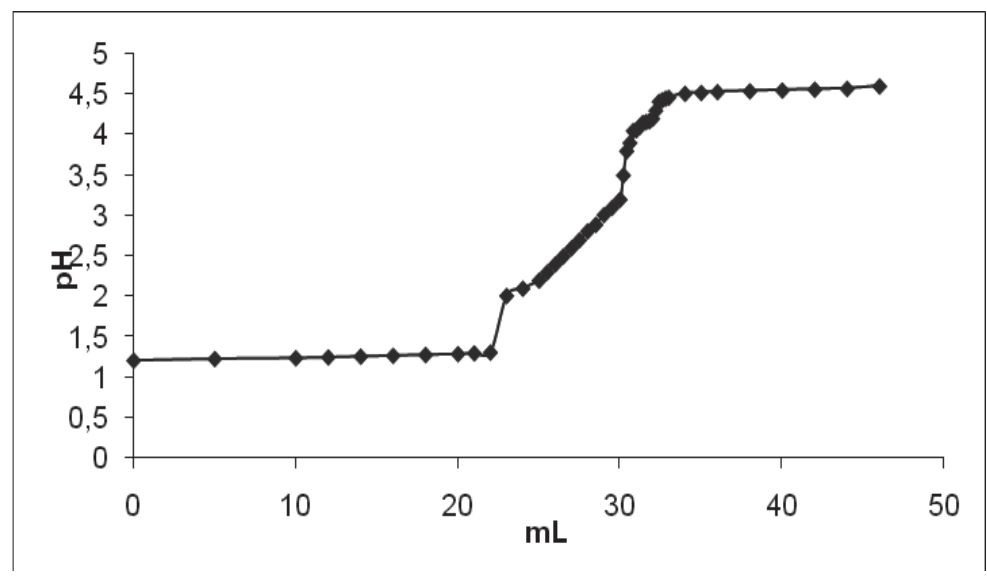

Jaiba

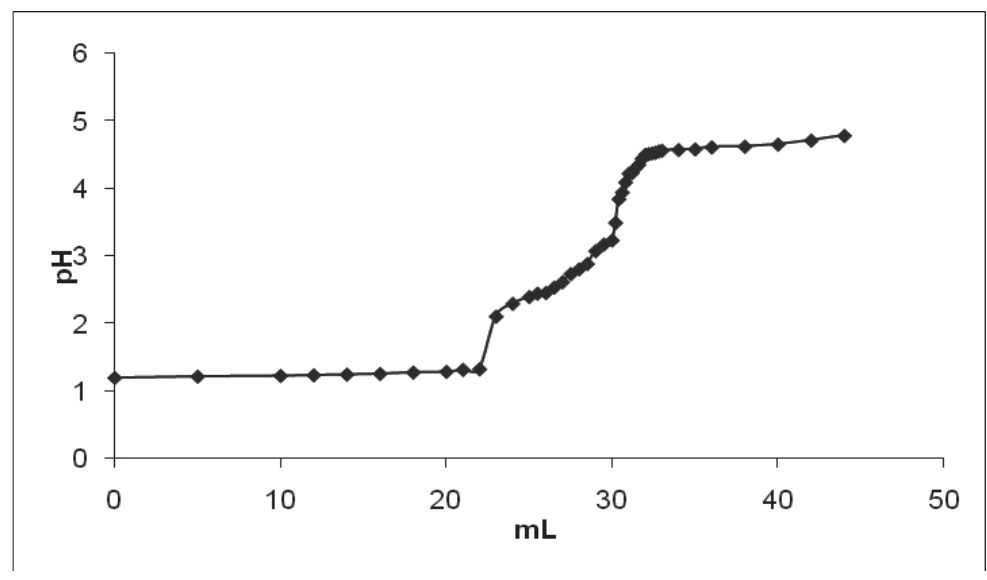

Camarón

Figura 2. Valoración potenciométrica de la quitina extraída de exoesqueleto.

Tabla 1. Resultados del análisis de las proporciones de grupos aminos en la muestra de jaiba, camarón y estándar, se presenta la media de tres experimentos.

\begin{tabular}{|c|c|c|c|c|c|}
\hline MUESTRA & $\mathrm{Y}(\mathrm{mL})$ & $\mathrm{X}(\mathrm{mL})$ & $(\mathrm{Y}-\mathrm{X}) \mathrm{mL}$ & $\% \mathrm{NH} 2$ & $\begin{array}{c}\text { GRADO DE } \\
\text { ACETILACION }\end{array}$ \\
\hline QUITINA JAIBA & 30,2 & 22 & 8,2 & 24,76 & $75,078 \%$ \\
\hline $\begin{array}{c}\text { QUITINA } \\
\text { CAMARÓN }\end{array}$ & 30 & 22 & 8 & 24,15 & $74,97 \%$ \\
\hline $\begin{array}{c}\text { QUITINA } \\
\text { ESTANDAR }\end{array}$ & $78,45 *$ & $56,71 *$ & $21,74 *$ & 25,90 & $74,978 \%$ \\
\hline
\end{tabular}

* Dato suministrado por el proveedor. 
Al analizar la tabla 2, se observa que las muestras de quitina en camarón y jaiba presentan un bajo porcentaje de humedad, debido a que la estructura de la quitina presenta sus grupos acoplados y no permite la inclusión de agua, dando un compuesto acétilado y con una alta pureza, comparada con la quitina comercial, cuyo porcentaje de humedad máximo es de un $10 \%$.

En cuanto al residuo de ignición, se observó que el porcentaje de ceniza en la quitina de jaiba $(0,61 \%)$ es más alto con respecto al porcentaje de ceniza de la quitina de camarón (0,09\%); lo que conduce, a un contenido mayor de minerales en la estructura de la jaiba y menor en el camarón, indicando que el proceso de desmineralización fue más alto en el camarón $(11,6 \%)$ que en la jaiba $(6,6 \%)$, lo cual se refleja en la cantidad de minerales obtenidos, como calcio y magnesio, establecidos en la tabla 2.

Se evidenciam, igualmente, el aumento de minerales, como calcio y magnesio de la quitina de jaiba, con respecto a la quitina de camarón, lo que corrobora el proceso de desmineralización, descrito por Bough et al. (1978), para camarón molido y tamizado y no para jaiba, deduciendo que hay que aumentar el tiempo de retención de la muestra de jaiba en el proceso de desmineralización y hacer la modificación de la técnica, en la obtención de quitina para este crustáceo.

Igualmente, se observa un aumento en el porcentaje de grasa de la quitina de camarón, respecto a la quitina extraída de la jaiba, lo que indica que el procedimiento aplicado y descrito por Bough et al. (1978) es aceptable para ambos casos.
La tabla 2 establece los porcentajes de nitrógeno y proteínas encontrados en la quitina extraída de la caparazón de jaiba y camarón, lo cual, indica que son muy similares a los encontrados en el estándar de quitina, que corresponde con los porcentajes encontrados por otros autores (Abdou et al. 2008; Khorrami et al. 2012) y en los que el proceso de desproteinización se realizó de forma eficiente, teniendo en cuenta las diferencias en las estructuras de cada caparazón (Mathur et al. 1990).

Una vez evaluado el t-student descrito en la tabla 3 de la quitina extraída del caparazón de jaiba y camarón, se procedió a realizar una comparación con lo descrito por los autores, con el t-tabulado, para un nivel de confianza del $95 \%$, determinando, en uno de sus valores, el aumento de cenizas en la quitina extraída del caparazón de la jaiba, que influye de manera significativa en los cálculos propuestos y, por consiguiente, en la prueba estadística. A diferencia de los demás parámetros estudiados no mostraron un comportamiento fuera de la tendencia planteada por los diferentes autores consultados.

De acuerdo a las propiedades fisicoquímicas de la quitina obtenida es potencialmente útil a nivel industrial. Debido a su alto contenido proteico esta puede ser utilizada para alimentación animal.

Los espectros IR presentan concordancia en las bandas de quitina del estándar y la quitina extraída del caparazón de jaiba y camarón. El acople de los grupos en la estructura de la quitina, le permite presentar bajos porcentajes de humedad, evitando así la inclusión de agua, originando un compuesto acetilado y de alta pureza.

Tabla 2. Composición de las muestras de quitina comercial, de jaiba y camarón.

\begin{tabular}{|l|c|c|c|}
\hline \multicolumn{1}{|c|}{ Composición (\%) } & Quitina de jaiba & Quitina de camarón & Quitina estándar \\
\hline Humedad & 5,97 & 5,60 & 2 a $10^{*}$ \\
\hline Nitrógeno & 2,95 & 4,73 & 3.30 a $8,50^{*}$ \\
\hline Cenizas & 0,610 & 0.093 & $0,270^{*}$ \\
\hline Grasa & 0,1626 & 0,7933 & 0 a $14^{*}$ \\
\hline Proteína & 18,44 & 29,594 & 20 a $40^{*}$ \\
\hline Calcio(mg) & 215,5 & 73,5 & NR \\
\hline Magnesio(mg) & 7,089 & 4,251 & NR \\
\hline Solidos totales & 94,03 & 94,40 & 90 a $98^{*}$ \\
\hline Residuos de lgnición & 0,6090 & 0,0930 & $0,2700^{*}$ \\
\hline
\end{tabular}

*Datos suministrados por el proveedor. 
Tabla 3. Comparación de la $t$-student de la quitina extraída.

\begin{tabular}{|c|c|c|}
\hline CARACTERÍSTICAS & t- calculado & t- tabulado \\
\hline Jaiba & & \multirow{5}{*}{4,30} \\
\hline Cenizas & 52,203 & \\
\hline Grasa & $-325,63$ & \\
\hline Nitrógeno & $-97,490$ & \\
\hline Proteínas & $-58,10$ & \\
\hline \multicolumn{3}{|l|}{ Camarón } \\
\hline Cenizas & 1,028 & \multirow{4}{*}{4,30} \\
\hline Grasa & $-276,32$ & \\
\hline Nitrógeno & $-13,605$ & \\
\hline Proteínas & $-96,789$ & \\
\hline
\end{tabular}

De la valoración potenciométrica en la muestra de quitina extraída del caparazón de jaiba y camarón, se puede establecer que las gráficas obtenidas tienen un comportamiento similar, representado en una curva sigmoidea, para ambos casos. Los valores de grasa, de nitrógeno y de proteínas en las muestras de quitina obtenida del caparazón de jaiba y camarón, se encuentran dentro de los límites aceptados, con respecto a las comparaciones realizadas por los autores propuestos. Se puede afirmar que los desechos de los crustáceos son aptos para ser procesados y obtener quitina que, por sus múltiples propiedades, puede llegar a ser comercializada.

Conflictos de intereses: El manuscrito fue preparado y revisado con la participación de todos los autores, quienes declaramos que no existe conflicto de intereses que ponga en riesgo la validez de los resultados presentados.

\section{BIBLIOGRAFÍA}

1. A.O.A.C. 2005. Official Methods of Analysis of the AOAC International. 18th ed. Horowitz, W.; Latimer Jr., G.W. (eds) Association of Analytical Chemists. Maryland. United States. 780p.

2. ABDOU, E.S.; NAGY, K.S.A.; ELSABE, M.Z. 2008. Extraction and characterization of chitin and chitosan from local sources. Bioresource Technology 99:1359-1367.

3. AL-SAGHEER, F.A.; AL-SUGHAYER, M.A.; MUSLIM, S; ELSABEE, M.Z. 2009. Extraction and characterization of chitin and chitosan from marine sources in the Persian Gulf. Carbohydr. Polymers. 77:410-419.

4. BOUGH, W.A.; SALTER, W.L.; WU, A.C.; PERKINS, B.E. 1978. Influence of manufacturing variables on the characteristics and effectiveness of chitosan products. I. Chemical composition, viscosity and molecular weight distribution of chitosan products. Biotechn. Bioeng. 20(12):1931-1943.

5. BRUGNEROTTO, J.; LIZARDI, J.; GOYCOOLEA, F.M.; ARGÜELLES-MONAL, W.; DESBRIÈRES, J.; RINAUD, M. 2001. An infrared investigation in relation with chitin and chitosan characterization. Polymer. 42:3569-3580.

6. CAHÚ, T.B.; SANTOS, S.D.; MENDES, A.; CÓRDULA, C.R.; CHAVANTE, S.F.; CARVALHO JR. L.B.; NADER, H.B.; BEZERRA, R.S. 2012. Recovery of protein, chitin, carotenoids and glycosaminoglycans from Pacific white shrimp (Litopenaeus vannamei) processing waste. Process Biochem. 47:570-577.

7. DOMSZY, J.; ROBERTS, G. 1985. Evaluation of infrared spectroscopic techniques for analyzing chitosan. Makromol. Chem. 186:1671-1677.

8. GOODRICH, J.D.; WINTER, W.T. 2007. $\alpha$-Chitin nanocrystals prepared from shrimp shells and their specific surface area measurement. Biomacromolec. 8:252-257.

9. HERNÁNDEZ, H.; ÁGUILA, E.; FLORES, O.; VIVEROS, E.L.; RAMOS, E. 2009. Obtención y caracterización de quitosano a partir de exoesqueletos de camarón. Superficies y Vacío. 22(3):57-60.

10. HIDALGO, C.; SUÁREZ, Y.; FERNÁNDEZ, M. 2008. Validación de una técnica potenciométrica para determinar el grado de desacetilación. Ars Pharm. 49(3):245-257. 
11. JIANG, X.; CHEN, L.; ZHONG, W. 2003. A new linear potentiometric titration method for the determination of deacetylation degree of chitosan. Carbohydr. Polymers. 54:457-463.

12. KHORRAMI, K.; NAJAFPOUR, G.; YOUNESI, H.; HOSSEINPOURA, M. 2012. Production of Chitin and Chitosan from Shrimp Shell in BatchCultureof Lactobacillus plantarum. Chem. Biochem. Eng. Q. 26(3)217-223.

13. MASUM, S.; MONARUL, I.; MAHBUBUR, M; ASHRAFUL ISLAM MOLLA, A.; SHAIKH, S.K. 2011. Preparation of Chitosan from Shrimp Shell and Investigation of Its Properties. Int. J. Basic \& Applied Sci. IJBASIJENS. 11(1):77-80.

14. MATHUR, N.K.; NARANG, C.K. 1990. Chitin and chitosan, versatile polysaccharides from marine animals. J. Chem. Ed. 67(11):938-942.

15. MEYER, S.P.; BLIGH, D. 1981. Characterization of astaxanthin pigments from heat processed crawfish waste. J. Agric. Food Chem. 29(3):505-508.
16. MOHAMMED, M.H.; WILLIAMS, P.A.; TVEREZOVSKAYA, O. 2013. Extraction of chitin from prawn shells and conversion to low molecular mass chitosan. Food Hydrocoll. 31:166-171.

17. RØDDE, R.H.; EINBU, A.; VÂRUM, K.M. 2008. A seasonal study of the chemical composition and chitin quality of shrimp shells obtained from northern shrimp (Pandalus borealis). Carbohydrate Polymers 71(3):388-393.

18. RUIZ, A.I.; CARDELLE-COBAS, A.; GARCÍA-BERMEJO, A.B.; MONTILLA, A.; OLANO, A.; CORZO, N. 2013. Synthesis, characterization and functional properties of galactosylated derivatives of chitosan through amide formation.Food Hydrocoll. 33:245-255.

19. TELI, M.D; SHEIKH, J. 2012. Extraction of chitosan from shrimp shells waste and application in antibacterial finishing of bamboo rayon. Int. J. Biol. Macromol. 50:1195-1200.

Recibido: Julio 18 de 2014

Aceptado: Febrero 17 de 2015

Cómo citar:

Barros, I.; Guzmán, L.; Tarón, A. 2015. Extracción y comparación de la quitina obtenida a partir del caparazón de Callinectes sapidus y Penaeus vannameis. Rev. U.D.C.A Act. \& Div. Cient. 18(2): 227-234. 